\title{
Adaptability of the Logistics System in National Economic Mobilization Based on Blocking Flow Theory
}

\author{
Xiangyuan Jing, Qingmei Tan, Liusan Wu, and Xiaohui Li \\ School of Economics and Management, Nanjing University of Aeronautics and Astronautics, Nanjing 211106, China \\ Correspondence should be addressed to Liusan Wu; wuhusheng563520@126.com
}

Received 20 December 2013; Revised 7 April 2014; Accepted 9 April 2014; Published 28 April 2014

Academic Editor: Hamid Reza Karimi

Copyright ( 2014 Xiangyuan Jing et al. This is an open access article distributed under the Creative Commons Attribution License, which permits unrestricted use, distribution, and reproduction in any medium, provided the original work is properly cited.

\begin{abstract}
In the process of national economic mobilization, the logistics system usually suffers from negative impact and/or threats of such emergency events as wars and accidents, which implies that adaptability of the logistics system directly determines realization of economic mobilization. And where the real-time rescue operation is concerned, heavy traffic congestion is likely to cause a great loss of or damage to human beings and their properties. To deal with this situation, this article constructs a blocking-resistance optimum model and an optimum restructuring model based on blocking flow theories, of which both are illustrated by numerical cases and compared in characteristics and application. The design of these two models is expected to eliminate or alleviate the congestion situation occurring in the logistics system, thus effectively enhancing its adaptability in the national economic mobilization process.
\end{abstract}

\section{Introduction}

Mobilization of national economy refers to a series of activities of planning and organizing a nation's economy from the previously regular situation to an irregular state in order to satisfy emergency needs when wars or accidents occur [1]. In the whole operation, resources mobilization is given priority and thus the logistics activities play an essential role in the phases of resource preparation, distribution, and demobilization [2]. Since national economic mobilization is featured by quick response to wars and other urgent events, a logistics system that works mainly to meet emergency demand is defined as the national economic mobilization logistics (abbreviated as mobilization logistics). Specifically, the national economic mobilization logistics activities involve a whole procedure of spatially transferring mobilized resources from suppliers to end users through preparation, transportation, packaging, storage, and even consumption, and so forth [3].

Wars and emergency events, on the one hand, are the reasons why mobilization logistics comes into being and are the objects that the logistics system has to deal with, and, on the other hand, they actually form attacks and threats to the mobilization logistics [4]. As statistical data show, in the rescue operation aftermath a natural disaster, an ineffective logistics distribution usually causes substantial losses of casualties and property, accounting for approximately $15 \%$ to $20 \%$ of the overall losses [4]. Once suffering from an impact or threat, the mobilization logistics system is needed to adjust its own internal components and structural functions in order to become adaptable to any change, which is regarded as the logistics system's adaptability [5]. By referring to system disturbance and monitoring theories, the authors define the mobilization logistics system adaptability as an ability of a logistics system to respond to disturbance and emergency events such as wars and accidents through restructure of its internal elements and functions when the nation's mobilization logistics system is disturbed [6].

National economic mobilization logistics is essentially a summit logistics. Accordingly, if any war or unexpected event occurs, logistics demand will soar and logistics network systems will probably be attacked. Under the circumstances, there will emerge blocks in logistics network nodes and links and even a blocking in the whole network [7]. To achieve a higher capability to control the network in real time, some methodologies have been directly assigned to distributed flow objects [8-11]. In this paper, the authors reckon the blocking phenomenon to be coincident with issues described 
in blocking flow theories. Furthermore, considering potential losses incurred by serious traffic blocking, this paper attempts to construct a blocking-resistance optimum model and an optimum restructuring model using blocking flow theories, followed by a detailed analysis of their difference and usage. Such design is expected to eliminate or alleviate congestion situation occurring in the logistics system, thus effectively enhancing its adaptability in the national economic mobilization process.

\section{Generalization of Blocking Flow}

The concept of blocking flow originates from the maximum flow algorithm proposed by Dinitz in 1970, which was originally used to deal with the transition value in maximum flow algorithm; later on, it had been widely introduced in maximum flow algorithm. Since 1994, Professor Ning Xuanxi has been engaged in the blocking flow theory and application systematically and deeply, funded by the National Natural Science Foundation three times. He made great contributions to the blocking flow theory by having proposed relevant concepts and definitions, proved fundamental theorems, solved minimum flow problems in the network, constructed a basic frame of block theory, and made a series of progress in approaches and application [12].

\subsection{Basic Concepts of Network Flow}

(1) Value of flow: the materials' quantity passes through the arc $i$ - $j$ during a specific period of time, denoted by $f_{i j}$, a variable ready to be calculated in the network flow problem. The total flow value in a network is represented by $v(f)$.

(2) Capacity: the maximum permissible flow of an arc is generally denoted by $c_{i j}$.

(3) Feasible flow: the flow satisfying the conditions is as follows.

(a) Capacity constraint: As for each $\operatorname{arc} v_{i} v_{j} \in A$ (A standing for the arcs set), there is $0 \leq f_{i j} \leq c_{i j}$, that is, the flow in arc $i$ - $j$ being not more than its capacity.

(b) Equilibrium condition: The flow entering the initial vertex $v_{s}$ is equal to the total sum of flow, and the one outflowing from the terminal vertex $v_{t}$ is equal to total sum of flow, and the one entering an intermediate vertex $i(i \neq s, t)$ is equal to that outflowing this vertex, shown as

$$
\begin{aligned}
& \sum_{v_{s} v_{j} \in A} f_{s j}-\sum_{v_{j} v_{s} \in A} f_{j s}=v(f), \\
& \sum_{v_{t} v_{j} \in A} f_{t j}-\sum_{v_{j} v_{t} \in A} f_{j t}=-v(f), \\
& \sum_{v_{i} v_{j} \in A} f_{i j}-\sum_{v_{j} v_{i} \in A} f_{j i}=0 .
\end{aligned}
$$

(4) Saturated arc and unsaturated arc: The arc with the same flow value as the capacity (i.e., $f_{i j}=c_{i j}$ ) is called saturated arc, while that with less flow value than capacity (i.e., $f_{i j}<c_{i j}$ ) is called unsaturated arc.

(5) Forward arc and backward arc: In the complete network, the arc with direction from the initial vertex $v_{s}$ to the terminal vertex $v_{t}$ is called forward arcs, and the one with direction from $v_{t}$ to $v_{s}$ is called backward arc.

\subsection{Definitions of Blocking Flow}

Definition 1. An augrementing directed path from source vertex $s$ to sink vertex $t$ is defined as a path along which flow value can be increased and each arc in the path has the direction consistent with the flow direction. The augrementing directed path in discussion exists without repeated vertexes or arcs.

Definition 2. A feasible flow is defined as the saturated flow of the network if there is no augrementing directed path.

Definition 3. The saturated flow of a network is defined as the blocking flow if the value of the saturated flow is less than the entrance flow capacity of the network.

Definition 4 . The capacity potential at the vertex $V_{i}$ refers to the difference between the total capacities of outgoing arcs and the total capacity of incoming arcs at $V_{i}$, shown as

$$
\Phi_{v i}=\sum_{j} c_{i j}-\sum_{j} c_{j i},
$$

where $c_{j i}$ denotes the capacity of the arc $e_{j i}$. Clearly, when $\Phi_{A} \geq 0$, there will be no structural blocking phenomenon at the vertex $A$ (neither source vertex nor sink vertex); and, when $\Phi_{A}<0$, structural blocking will probably occur at the vertex $A$. In this case, the vertex with negative capacity potential is called structurally blocking vertex.

Definition 5. If capacities of all vertexes in a network (excluding source and sink vertexes) are commonly zero, such a network is called a totally balanced one.

\section{Blocking-Resistance Optimum Model for the Traffic Network}

Since the traffic network design is generally required not only to meet the demand for traffic flow but also to alleviate blocking phenomena as much as possible, both parameters of maximum flow and minimum flow in a network are worth special notice. Traditionally, some designed networks were unable to meet the demand for the maximum flow in practical operation by ignoring the minimum flow. Accordingly, to avoid any blocking in a network, the proposed model amends the original capacity parameters in a permissible range and thus makes the capacity potential of all vertexes more than zero, with exception of the source and sink vertex. 
3.1. Model Design. Take a traffic network $N=(V, A, s, t)$ into consideration, in which $s$ denotes the source vertex, $t$ the sink vertex, $V$ the vertex set, and $A$ the arc set. The original design is described as follows.

(1) The originally designed capacity, and the permissible maximum and minimum capacity are already known.

(2) The requirement concerning incoming flow is given.

(3) The cost used to construct one unit capacity of an arc is learned.

Note that, in the proposed model, the fluctuation of capacities corresponds to the original ones comparatively. The cost occurring from a unit capacity fluctuation of an arc is supposed to be equal, denoted as $b\left(e_{i j}\right)$ for the arc $e_{i j}$. The optimum objective is to achieve the least transformation cost [13].

The objective function is

$$
\min \left(\sum\left\{b\left(e_{i j}\right) \cdot \Delta c\left(e_{i j}\right) \mid e_{i j} \in E\right\}\right),
$$

where $\Delta c\left(e_{i j}\right)$ indicates the increased capacity of the arc $e_{i j}$.

The constraint conditions are shown below.

(1) As for the negative capacity potential, there is

$$
\begin{aligned}
\widetilde{\Phi}_{v_{i}}= & \sum_{j}\left\{\Delta c\left(e_{i j}\right) \mid e_{i j} \in E\right\} \\
& -\sum_{j}\left\{\Delta c\left(e_{j i}\right) \mid e_{j i} \in E\right\}+\Phi_{v_{i}}=0, \quad \forall v_{i} \in V .
\end{aligned}
$$

(2) As for the nonnegative capacity potential, there is

$$
\begin{aligned}
-\Phi_{v_{i}} \leq & \sum_{j}\left\{\Delta c\left(e_{i j}\right) \mid e_{i j} \in E\right\} \\
& -\sum_{j}\left\{\Delta c\left(e_{j i}\right) \mid e_{j i} \in E\right\} \leq 0, \quad \forall v_{i} \in V .
\end{aligned}
$$

(3) The incoming flow is restricted by

$$
\sum_{j} \tilde{c}\left(e_{s j}\right)=F, \quad \forall v_{i} \in V
$$

(4) In the network, each arc's flow value and capability are restricted by

$$
\begin{gathered}
x\left(e_{i j}\right) \leq \widetilde{c}\left(e_{i j}\right), \quad \forall e_{i j} \in E \\
m c_{2}\left(e_{i j}\right) \leq \tilde{c}\left(e_{i j}\right) \leq m c_{1}\left(e_{i j}\right), \quad \forall e_{i j} \in E
\end{gathered}
$$

where $m c_{1}\left(e_{i j}\right)$ and $m c_{2}\left(e_{i j}\right)$ are the maximum and minimum capacities, respectively.

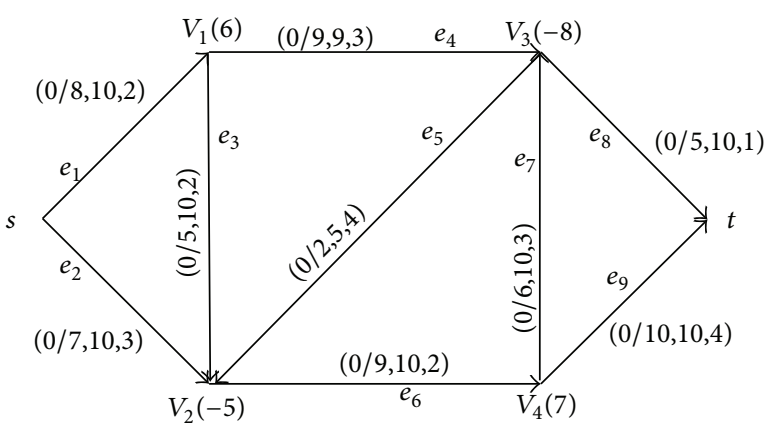

FIgURE 1: Numerical case of blocking-resistant optimum design.

3.2. Numerical Case. Figure 1 illustrates a traffic network related to the mobilization logistics system and six vertexes that represent six cities, respectively. As for each arc, there are four parameters: $x\left(e_{i}\right) / c\left(e_{i}\right), m c_{1}\left(e_{i}\right)$, and $b\left(e_{i}\right)$, of which $c\left(e_{i}\right)$ indicates the original capacity of the $\operatorname{arc} e_{i}$ with $m c_{2}\left(e_{i}\right)=$ 2 and $b\left(e_{i}\right)$ indicates the cost occurring from changing per unit capacity, and the numbers next to the vertex indicates the capacity potential of each vertex. Besides, the incoming flow is assumed to obey the rule of $F=15$. follows.

The blocking-resistant optimum design model is set up as

The objective function is

$$
\min \left(\sum\left\{b\left(e_{i}\right) \cdot \Delta c\left(e_{i}\right) \mid \forall e_{i} \in E\right\}\right)
$$

Constraint conditions are

$$
\begin{gathered}
-6 \leq \Delta c\left(e_{3}\right)+\Delta c\left(e_{4}\right)-\Delta c\left(e_{1}\right) \leq 0, \\
-7 \leq \Delta c\left(e_{7}\right)+\Delta c\left(e_{9}\right)-\Delta c\left(e_{6}\right) \leq 0, \\
\Delta c\left(e_{6}\right)-\Delta c\left(e_{2}\right)-\Delta c\left(e_{3}\right)-\Delta c\left(e_{5}\right)=0, \\
\Delta c\left(e_{5}\right)+\Delta c\left(e_{8}\right)-\Delta c\left(e_{4}\right)-\Delta c\left(e_{7}\right)=0, \\
\tilde{c}\left(e_{1}\right)+\tilde{c}\left(e_{2}\right)=15,
\end{gathered}
$$

$$
m c_{2}\left(e_{i}\right) \leq \Delta c\left(e_{i}\right)+c\left(e_{i}\right) \leq m c_{1}\left(e_{i}\right), \quad \forall e_{i} \in E
$$

Among them, formulas (9) and (10) separately correspond to the vertexes $v_{1}$ and $v_{4}$ who have positive capacity potentials, while formulas (11) and (12) separately correspond to the vertexes $v_{2}$ and $v_{3}$ who have negative capacity potentials. In addition, in formula (13), $e_{1}$ and $e_{2}$ indicate incoming arcs, and the incoming flow of network is specified as 15 . Let $\Delta c\left(e_{i}\right)=x_{i}$; then the matrix in terms of the above-mentioned objective function and constraint conditions are illustrated as follows.

The objective function is $\min (A X)$.

The constraint condition is $C \leq B X \leq D$, 
TABLE 1: Parameters comparison between the original design and the optimum design.

\begin{tabular}{lcccc}
\hline & Arcs capacity distribution & Maximum flow & Minimum saturated flow & Construction cost \\
\hline Original & $(8,7,5,9,2,9,6,5,10)$ & 14 & 9 & 163 \\
Optimum & $(10,5,2,8,2,9,2,8,7)$ & 15 & 15 & 131 \\
\hline
\end{tabular}

where

$$
\begin{aligned}
& A=\left[\begin{array}{lllllllll}
2 & 3 & 2 & 3 & 4 & 2 & 3 & 1 & 4
\end{array}\right] \\
& X=\left[\begin{array}{lllllllll}
x_{1} & x_{2} & x_{3} & x_{4} & x_{5} & x_{6} & x_{7} & x_{8} & x_{9}
\end{array}\right]^{T} \text {, } \\
& B=\left[\begin{array}{ccccccccc}
-1 & 0 & 1 & 1 & 0 & 0 & 0 & 0 & 0 \\
0 & 0 & 0 & 0 & 0 & -1 & 1 & 0 & 1 \\
0 & -1 & -1 & 0 & -1 & 1 & 0 & 0 & 0 \\
0 & 0 & 0 & -1 & 1 & 0 & -1 & 1 & 0 \\
1 & 1 & 0 & 0 & 0 & 0 & 0 & 0 & 0 \\
1 & 0 & 0 & 0 & 0 & 0 & 0 & 0 & 0 \\
0 & 1 & 0 & 0 & 0 & 0 & 0 & 0 & 0 \\
0 & 0 & 1 & 0 & 0 & 0 & 0 & 0 & 0 \\
0 & 0 & 0 & 1 & 0 & 0 & 0 & 0 & 0 \\
0 & 0 & 0 & 0 & 1 & 0 & 0 & 0 & 0 \\
0 & 0 & 0 & 0 & 0 & 1 & 0 & 0 & 0 \\
0 & 0 & 0 & 0 & 0 & 0 & 1 & 0 & 0 \\
0 & 0 & 0 & 0 & 0 & 0 & 0 & 1 & 0 \\
0 & 0 & 0 & 0 & 0 & 0 & 0 & 0 & 1
\end{array}\right], \\
& C=\left[\begin{array}{llllllllllllll}
-6 & -7 & 5 & 8 & 0 & -6 & -5 & -3 & -7 & 0 & -7 & -4 & -3 & -8
\end{array}\right]^{T} \text {, } \\
& D=\left[\begin{array}{llllllllllllll}
0 & 0 & 5 & 8 & 0 & 2 & 3 & 5 & 0 & 3 & 1 & 4 & 5 & 0
\end{array}\right]^{T} .
\end{aligned}
$$

The final objective value turns out to be -32 with the help of Lingo software. In other words, the transformation cost decreases by 32 units compared with the traditionally designed network. Table 1 shows the comparison of parameters between the newly designed network and the original one.

\section{The Blocking-Resistant Optimum Transformation Design for the Traffic Network}

This model is aimed at transforming the prevailing traffic system into a blocking-resistant one. Compared with the aforementioned one, this model appears to be different in existing arcs reconstruction, which requires expansion instead of reduction in capacity variation. As a result, structural blocks are diminished or reduced as many as possible, thus bringing about a totally balanced traffic network [14].

4.1. Model Construction. There are some conditions already known before the traffic network is reconstructed, including the following.

(1) The cost occurring from expanding unit capacity of each path (i.e., the length of each arc), denoted as $b_{i}$, is dependent on facilities removal charges, path's length, and operation complexity.
(2) The maximum capacity limit for each path is described as $m c_{i j}$.

(3) In addition, each path is required to be expanded instead of narrowed; that is, $\Delta c_{i j} \geq 0$.

As for each arc, given the four parameters of $\left[x_{i j}, c_{i j}, m c_{i j}\right.$, $b_{i j}$ ], among which $x_{i j}$ indicates the current flow volume, $c_{i j}$ the original capacity, $m c_{i j}$ the maximum capacity, and $b_{i j}$ the cost paid to improve unit capacity (if $x_{i j}<c_{i j}, b_{i j}=0$ ), blockingresistant transformation is thus to be understood as a problem of how to enlarge every arc's capacity with the least cost. That is, the negative capacity potential vertexes will be increased as many as possible, while the positive ones will be reduced and not less than 0 . After being transformed, the network will suffer less structural blocks with improvement in negative capacity potentials.

Let the added capacity value of each arc be

$$
\Delta c\left(e_{i j}\right)=\widetilde{c}\left(e_{i j}\right)-c\left(e_{i j}\right) .
$$

The objective function is

$$
\min \left(\sum\left\{b\left(e_{i j}\right) \cdot \Delta c\left(e_{i j}\right) \mid \Delta c\left(e_{i j}\right)>0, e_{i j} \in E\right\}\right)
$$

where $b\left(e_{i j}\right)$ refers to the cost needed for improving unit capacity of $\operatorname{arc} e_{i j}$.

The constraints are as follows.

(1) For the negative capacity potential vertex, there is

$\sum_{j}\left\{\Delta c\left(e_{i j}\right) \mid e_{i j} \in E\right\}-\sum_{j}\left\{\Delta c\left(e_{j i}\right) \mid e_{j i} \in E\right\} \geq 0$

(2) For the nonnegative capacity potential vertex, there is

$$
\begin{aligned}
-\Phi_{v_{i}} \leq & \sum_{j}\left\{\Delta c\left(e_{i j}\right) \mid e_{i j} \in E\right\} \\
& -\sum_{j}\left\{\Delta c\left(e_{j i}\right) \mid e_{j i} \in E\right\} \leq 0, \quad \forall v_{i} \in V .
\end{aligned}
$$

(3) Furthermore, restrictions on flow volume and capacity for the network are

$$
\begin{gathered}
x(e) \leq \tilde{c}(e), \quad \forall e \in E, \\
0 \leq \widetilde{c}(e) \leq m c(e), \quad \forall e \in E .
\end{gathered}
$$

Note that the incoming flow is limited to being $F$ and the maximum capacity of arc $e$ is $m c(e)$. 
TABLE 2: Comparison of arcs' capacity and saturated flow before and after transformation.

\begin{tabular}{lcc}
\hline & The network before transformation & The network after transformation \\
\hline Arcs' capacity distribution & {$[8,7,5,9,2,9,6,5,10]$} & {$[10,7,5,9,2,10,6,9,10]$} \\
Network's maximum flow & 14 & 17 \\
Network's minimum saturated flow & 9 & 13 \\
Minimum transformation cost & - & 10 \\
\hline
\end{tabular}

4.2. Numerical Case. Referring to Figure 1 , the figures above the arrow line represent $x_{i j} / c_{i j}, m c_{i j}$, and $b_{i j}$, and those next to all nodes describe the capacity potentials. Besides, the nodes $v_{2}, v_{3}$ are negative capacity potential vertexes, while $v_{1}, v_{4}$ are positive capacity potential vertexes. Before being transformed, the maximum flow and minimum flow are $F=$ 14 and $v=9$, respectively. The optimum design model is set up as below.

The objective function is

$$
\min \left(\sum\left\{b\left(e_{i}\right) \cdot \Delta c\left(e_{i}\right) \mid \forall e_{i} \in E\right\}\right) .
$$

The constraint conditions are

$$
\begin{gathered}
-6 \leq \Delta c\left(e_{3}\right)+\Delta c\left(e_{4}\right)-\Delta c\left(e_{1}\right) \leq 0, \\
-7 \leq \Delta c\left(e_{7}\right)+\Delta c\left(e_{9}\right)-\Delta c\left(e_{6}\right) \leq 0, \\
\Delta c\left(e_{6}\right)-\Delta c\left(e_{2}\right)-\Delta c\left(e_{3}\right)-\Delta c\left(e_{5}\right) \geq 0, \\
\Delta c\left(e_{5}\right)+\Delta c\left(e_{8}\right)-\Delta c\left(e_{4}\right)-\Delta c\left(e_{7}\right) \geq 0, \\
\widetilde{c}\left(e_{1}\right)+\widetilde{c}\left(e_{2}\right)=15, \\
m c_{2}\left(e_{i}\right) \leq \Delta c\left(e_{i}\right)+c\left(e_{i}\right) \leq m c_{1}\left(e_{i}\right), \quad \forall e_{i} \in E .
\end{gathered}
$$

We obtain the objective value of 10 using Lingo software. The optimized network is illustrated in Figure 2, and both the arc capacity and saturated flow before and after transformation are compared in Table 2.

\section{Conclusion}

In short, the traffic-network blocking-resistant optimum model differs from the blocking-resistant optimum transformation model in nature.

As far as the optimum design model is mentioned, the capacity parameters in original design need to be amended with the objective of preventing the network from structural blocks with the capacity potential being less than zero for each vertex, except for source and intermediate vertexes. As shown in the numerical case, once optimized, the capacity potential of vertexes $v_{2}, v_{3}$ with negative ones in original network becomes 0 and the maximum and minimum saturated flow are equal, thus resulting in a totally balanced network.

In the optimum transformation model, however, the objective is to diminish and reduce structural blocking vertexes as many as possible. Seen from Figure 2, through transformation, the vertex $v_{2}$ and vertex $v_{3}$ achieve the capacity potentials of -4 and -4 , compared with -5 and -8 without transformation, respectively. That is, the two vertexes

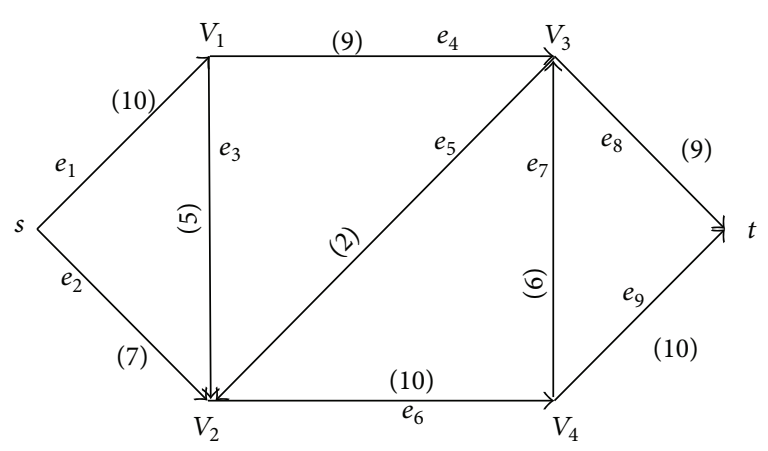

Figure 2: Transformed Network.

mentioned above still have negative capacity potentials, which maintain structural blocks in the network. In other words, the network is likely to suffer from blocking phenomena. More importantly, both the maximum and minimum saturated flow will increase simultaneously after experiencing transformation, and thus effectively improving the network.

In conclusion, the optimum model is suitable for the phase during which a traffic network is being designed, while the optimum transformation model is more adaptable for reconstruction of the already established traffic network. In mobilization logistics practice, the optimum transformation model will be commonly utilized for it helps prevent or diminish blocking phenomena by increasing arcs' capacity in accordance with actual conditions of traffic network transformation, which may lay a solid foundation for the research of mobilization logistics system adaptability.

In practice, travel time of saturated flows is one of the essential parameters employed in optimization and transformation designing process. Actually, the travel time in question seems ambiguous and uncertain owing to coeffect of environmental and human factors. Accordingly, to achieve a reasonable resource allocation, our further research will focus on a synthetic optimization combining reconstruction cost, travel time, and saturated flow distribution.

\section{Conflict of Interests}

The authors declare that there is no conflict of interests regarding the publication of this paper.

\section{References}

[1] Q. Zhu, National Economic Mobilization, Publishing House of Military Science, Beijing, China, 2nd edition, 2007. 
[2] J. Zhang, "Economic mobilization logistics problems," Jiangsu Business, no. 2, pp. 61-63, 2008.

[3] J. Cui and L. Kong, "The logistics chain of mobilization of national economy," Journal of Beijing Institute of Technology, vol. 6, no. 4, pp. 16-19, 2004.

[4] Q. Zhu, China's Economic Mobilization Adaptability, Publishing House of Military Science, Beijing, China, 2007.

[5] L. Shang and Q. Tan, "The connotation and characteristics of economic mobilization logistics network," China Circulation Economy, no. 7, pp. 25-28, 2013.

[6] J. Liu, National economy mobilization logistics system construction and the key technology [M.S. thesis], Nanjing University of Aeronautics \& Astronautics, 2013.

[7] X. Jing and Q. Tan, "The evaluation of national economy mobilization logistics system's adaptability," Economy of Technology, vol. 4, no. 2, pp. 24-28, 2013.

[8] A. Mehrsai, H.-R. Karimi, K.-D. Thoben, and B. Scholz-Reiter, "Using metaheuristic and fuzzy system for the optimization of material pull in a push-pull flow logistics network," Mathematical Problems in Engineering, vol. 2013, Article ID 359074, 19 pages, 2013.

[9] S. Dashkovskiy, H.-R. Karimi, and M. Kosmykov, "Stability analysis of logistics networks with time-delays," Journal of Production Planning \& Control, vol. 24, no. 7, pp. 567-574, 2013.

[10] S. Dashkovskiy, H.-R. Karimi, and M. Kosmykov, "A LyapunovRazumikhin approach for stability analysis of logistics networks with time-delays," International Journal of Systems Science, vol. 43, no. 5, pp. 845-853, 2012.

[11] H.-R. Karimi, N. A. Duffie, and S. Dashkovskiy, "Local capacity $H_{\infty}$ control for production networks of autonomous work systems with time-varying delays," IEEE Transactions on Automation Science and Engineering, vol. 7, no. 4, pp. 849-857, 2010.

[12] X. Ning, Flow Theory and Its Application, Science Press, Beijing, China, 2009.

[13] W. Wu and X. Ning, "Anti blockage transformation of an emergency evacuation network," Systems Engineering, vol. 21, no. 3, pp. 244-247, 2006.

[14] W. Wu and X. Ning, "Simulation research on improving the flow capability of emergency networks," Chinese Management Science, vol. 14, no. 3, pp. 86-90, 2006. 


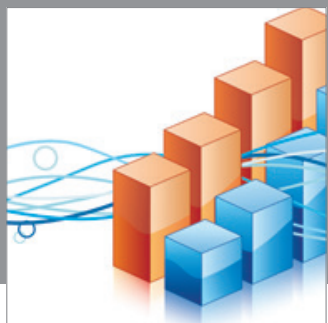

Advances in

Operations Research

mansans

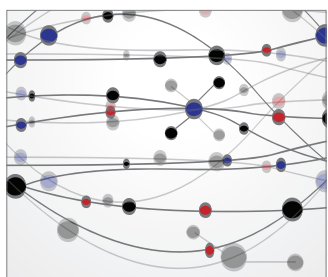

The Scientific World Journal
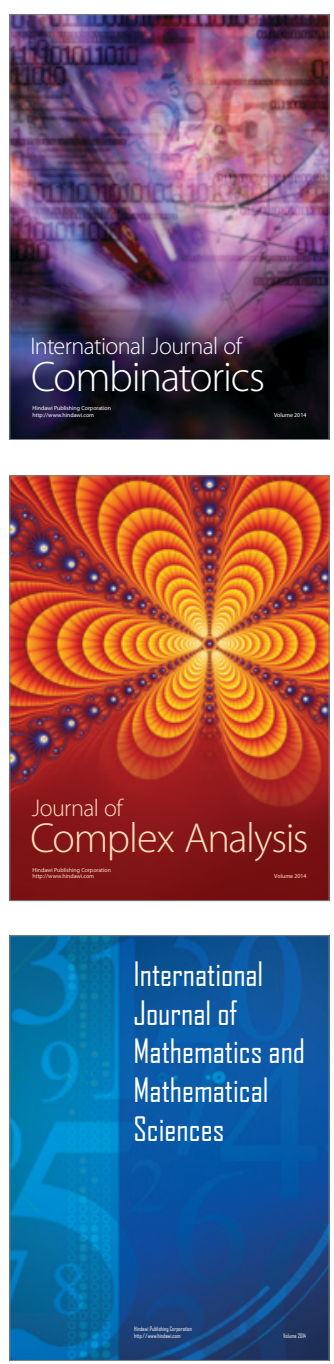
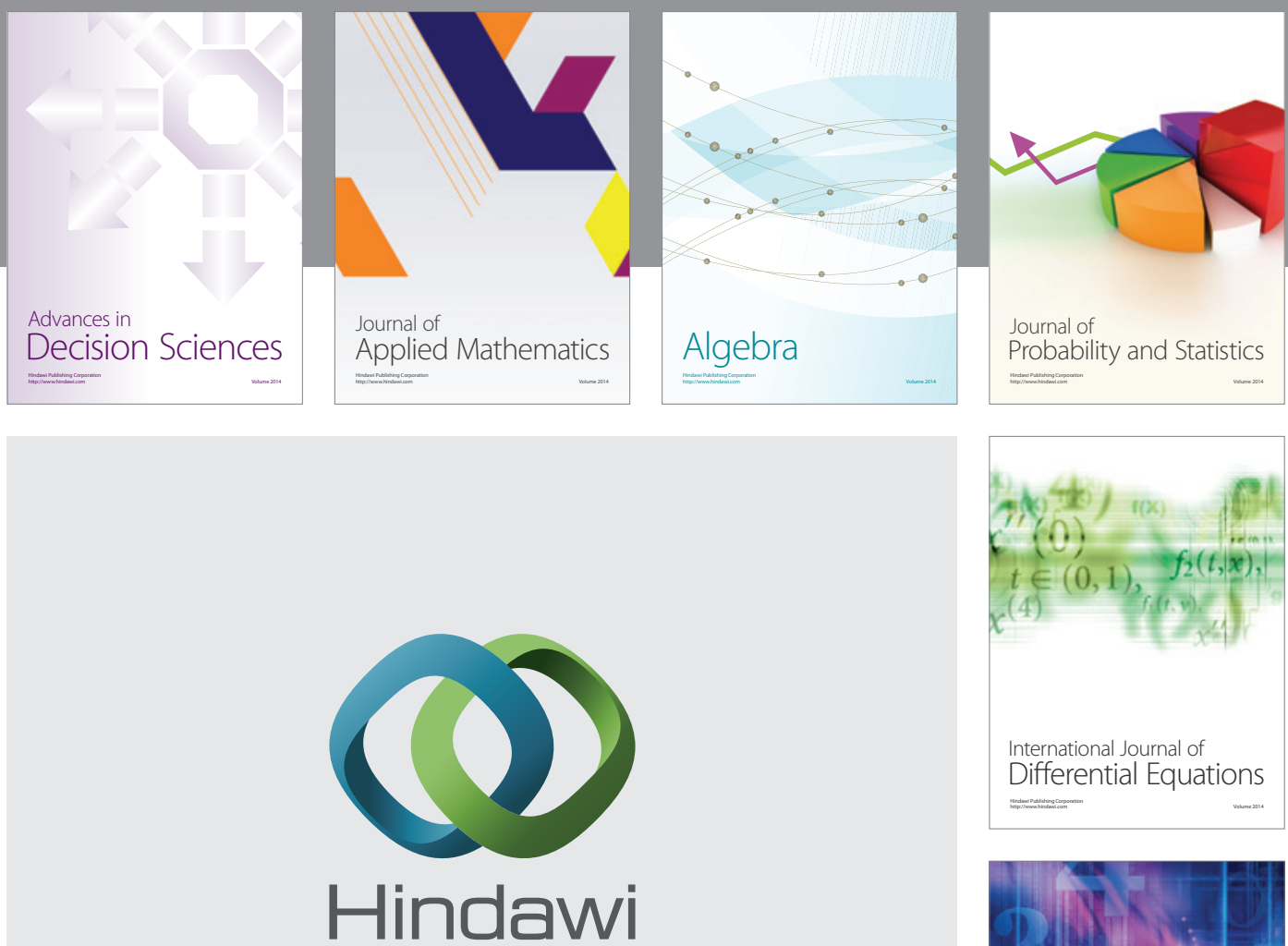

Submit your manuscripts at http://www.hindawi.com
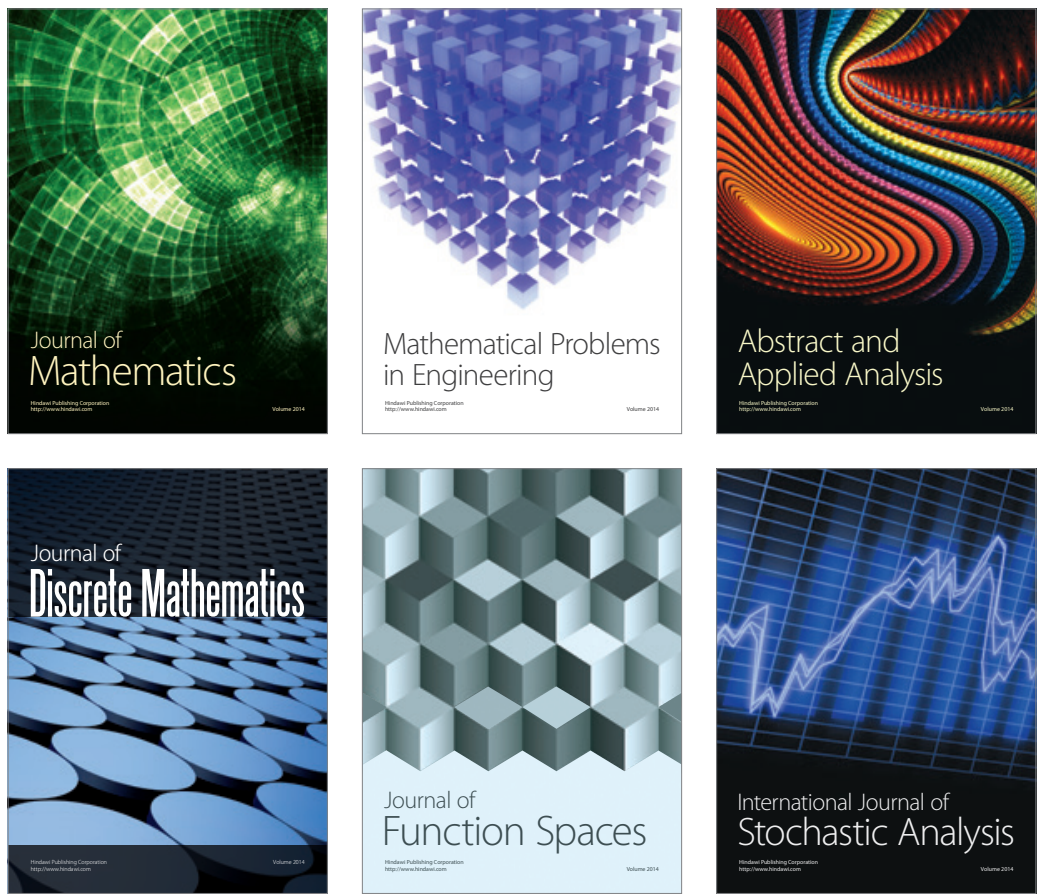

Journal of

Function Spaces

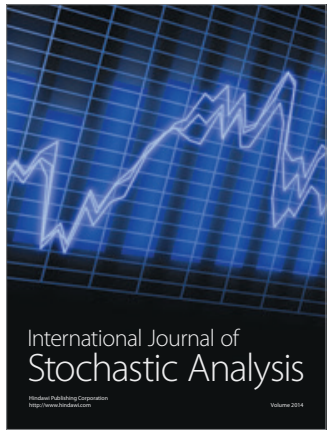

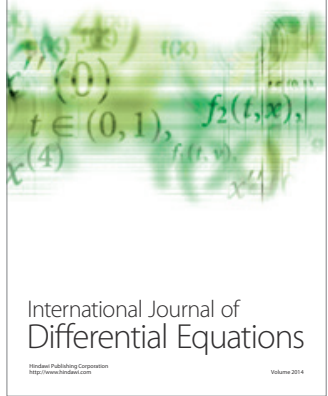
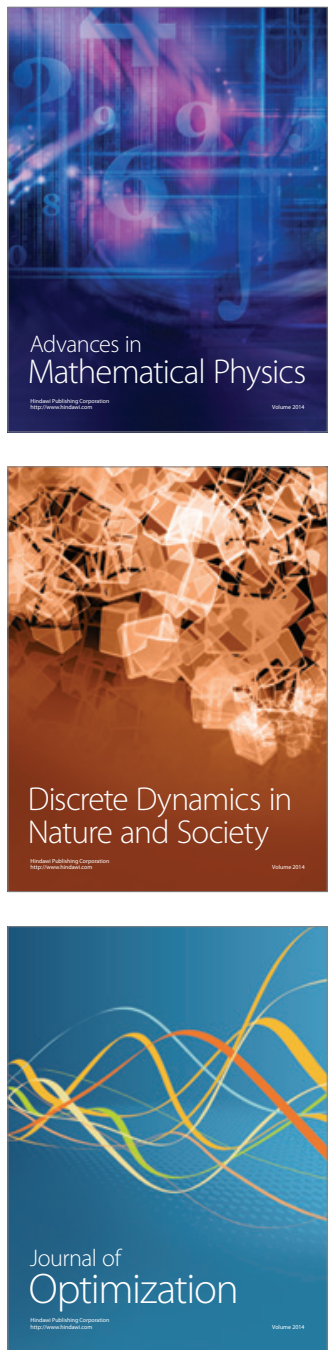